\title{
AUTOMATED CONTROL SYSTEMS
} АВТОМАТИЗИРОВАННЫЕ СИСТЕМЫ УПРАВЛЕНИЯ

\author{
УДК 621.377
}

\section{А.К. Иванов}

\section{МОДЕЛИРОВАНИЕ ПРОСТРАНСТВЕННОГО ПРЕОБРАЗОВАНИЯ ИНФОРМАЦИОННЫХ РЕСУРСОВ ОРГАНОВ УПРАВЛЕНИЯ}

\begin{abstract}
Иванов Александр Куприянович, доктор технических наук, окончил физический факультет Иркутского государственного университета, аспирантуру Московского высшего технического училища им. Н.Э. Баумана, докторантуру Ульяновского государственного технического университета. Главный научный сотрудник ФНПЦ АО «НПО «Марс». Имеет монографии, учебное пособие, статьи в области математического моделирования иерархических АСУ реального времени. [e-mail: mars@mv.ru].
\end{abstract}

\section{Аннотация}

Построены дифференциальные модели информационных процессов органов управления, учитывающие пространственное распределение и преобразование данных. Рассмотрены различные формы взаимодействия разнородных данных с диффузионным распространением от функций общего вида до уравнения Лотки. Описан порядок исследования моделей с линеаризацией в особых точках. Определены условия колебательных режимов и распространения информационных волн. Случай генерации данных в каждой точке органа управления по логистическому закону приведен к уравнению Фишера. Методами преобразования переменных и синус-преобразования решены уравнения диффузии с генерацией данных по экспоненциальному закону, уравнения диффузии с конвективным распространением данных и уравнения диффузии, в которых учтены генерация и конвективное распространение. Приближенные результаты представлены в графическом виде. Разработанная совокупность математических моделей направлена на повышение уровня автоматизации органов управления, установление оптимальной организации деятельности должностных лиц. Показана перспектива формализации процессов принятия решений.

Ключевые слова: органы управления, информационные процессы, математическое моделирование, системы дифференциальных уравнений.

doi:

\section{MODELING SPATIAL TRANSFORMATION OF INFORMATION RESOURCES OF MANAGEMENT BODIES}

Aleksandr Kupriianovich Ivanov, Doctor of Sciences in Engineering; graduated from the Faculty of Physics at Irkutsk State University, from the postgraduate studies at Bauman Moscow Technical School, from the Doctoral Studies at Ulyanovsk State Technical University; Chief Staff Scientist of FRPC JSC 'RPA 'Mars'; an author of monographs, text book, articles in the field of the mathematical modeling of hierarchical real-time computeraided control systems.e-mail: mars@mv.ru. 


\section{Abstract}

Differential models for information processes of management bodies are constructed, which take into consideration spatial distribution and data transformation. The various forms of heterogeneous data interaction with a diffusion propagation from a general function up to the Lotka-Volterra equation are considered. A procedure for researching models with a linearization at special points is described. Oscillatory conditions and information wave transmission are defined. The case of data generation at each point of management body at a logistic law is reduced to the Fisher equation. Diffusion equations with data generation at an exponential rate as well as diffusion equations with convective data propagation, and diffusion equations that take into consideration the generation and convective propagation are solved by methods of transformation of variables and sinus transformation. The approximate results are presented in graphical form. The developed set of mathematical models is aimed to improve the automation level of management bodies, to identify an optimal organization of officials' activity. The prospects for formalizing the decision-making procedure is demonstrated.

Key words: management bodies, information processes, mathematical modeling, differential equation systems.

\section{Введение}

В разработанных дифференциальных моделях информационных процессов органов управления можно выделить две части. Модели, описывающие различного рода преобразования данных без пространственного распределения, и модели пространственного распространения информационных ресурсов без их преобразования. Модели преобразования основаны на аналогии с задачами химической кинетики и популяции организмов в биологии [1]. Модели распространения связаны с задачами диффузии и теплопроводности $[2,3]$. Поиск аналогий и использование применяемого в других областях развитого математического аппарата обусловлен трудностями формального представления функций должностных лиц в органах управления и отсутствием целенаправленной работы по их исследованию. Следующим шагом будет построение объединенной модели распространения информационных ресурсов с преобразованием данных в каждой точке пространства. Аналогом станут дифференциальные уравнения, описывающие распределенные экологические системы с разной степенью взаимодействия элементов, а также уравнения диффузии с конвективным распространением вещества и обменом с внешней средой. В зависимости от детализации и точности представления реальных объектов находятся аналитические решения или проводятся качественные исследования. В [4] исследуется общее кинетическое уравнение взаимодействия элементов и их диффузии в пространстве. Система, в которой происходит взаимодействие нулевого, первого и второго порядков, рассматривается в [5], с исследованием задачи взаимодействия двух видов элементов. Конкретное взаимодействие двух видов, приводящее к уравнению Лотки, описано в [6]. Отсутствие взаимодействия, распространение одного вида с генерацией по логистическому закону приводит к уравнению Фишера [7]. Уравнения диффузии с конвекцией и обменом вещества с внешней средой по экспоненциальному закону относятся к математической физике. Известны методы решения таких уравнений при определенных граничных и начальных условиях [8]. Анализ информационных процессов органов управления показывает справедливость приведенных аналогий [1,
9, 10]. Орган управления представляет собой распределенный объект, в первом приближении одномерный, с последовательной цепочкой переработки данных должностными лицами. Поток входных сообщений определяет граничное условие. В каждой точке пространства органа управления производится обработка данных и формирование новых знаний, процесс приближенно можно описать логистическим или экспоненциальным законами. Распространение информации происходит по некоторому алгоритму (конвективным путем) или естественным образом от точки к точке за счет градиента концентрации ресурсов (диффузионным путем). В общем случае различные виды информационных ресурсов взаимодействуют между собой, оказывая взаимное влияние на рост концентрации и появление новых видов. Существенным моментом является творческий характер работы должностных лиц, который можно представить как накопление знаний и преодоление некоторого интеллектуального барьера [11].

\section{1 ОБЩИЕ СВЕДЕНИЯ О ДИНАМИЧЕСКИХ ПРОЦЕССАХ В РАСПРЕДЕЛЕННЫХ СИСТЕМАХ}

Повышение точности математических моделей органов управления достигается объединением и совместным рассмотрением различных информационных процессов. В ряде случаев можно использовать математический аппарат из других областей науки и техники, с определенной адаптацией под специфику задач управления. Сложность моделей и точность представления реальных объектов определяется задачами исследования.

По аналогии с экологической задачей, рассмотренной в [4], приведем описания информационных процессов в распределенном органе управления, в котором наряду с диффузионным переносом учитывается взаимодействие информационных ресурсов разного вида между собой.

Считаем, что $U_{i}(x, t)$ - объем информационных ресурсов (или количество документов) $i$-го вида в единице пространства органа управления, зависящий от времени $t$ и координаты $x$. Общие кинетические уравнения взаимодействия документов и их диффузии в пространстве имеют вид: 


$$
\frac{\partial U_{i}}{\partial t}=Q_{i}\left(U_{1}, \ldots, U_{n}, x\right)+D_{i} \frac{\partial^{2} U_{i}}{\partial x^{2}},
$$

где $D_{i}$ - коэффициенты диффузии;

$Q_{i}$ - функции взаимодействия между документами. Пусть $\bar{U}=\left\{\bar{U}_{1}, \bar{U}_{2}, \ldots, \bar{U}_{n}\right\}$ - стационарное, однородное по пространству решение точечной системы

$$
\frac{d U_{i}}{d t}=Q_{i}\left(\bar{U}_{1}, \ldots, \bar{U}_{n}\right)
$$

Для исследования устойчивости вводится малое возмущение относительно однородного решения

$U_{i}^{\prime}=U_{i}(x, t)-\bar{U}_{i}$.

Линеаризованная приведенная система будет иметь следующий вид:

$$
\begin{aligned}
& \quad \frac{\partial U_{i}^{\prime}}{\partial t}=\sum_{j=1}^{n} a_{i j} U_{j}^{\prime}+D_{i} \frac{\partial^{2} U_{i}^{\prime}}{\partial x^{2}}, \\
& \text { где } a_{i j}=\left(\frac{\partial Q_{i}}{\partial U_{i}^{\prime}}\right)_{U_{j}^{\prime}}=0 .
\end{aligned}
$$

Решение системы представляется в виде суперпозиции волн:

$$
U_{i}^{\prime}=a_{i} e^{p_{k} t+i k r},
$$

где $k$ - волновое число, определяющее длину волны $\lambda_{k}=2 \pi / k$.

Подставляя решение в линеаризованную систему и используя условие существования ее нетривиальных решений, получим дисперсионное уравнение, связывающее комплексные частоты $p_{k}=\delta_{k}+i w_{k}$, волновое число $k$ и коэффициенты системы

$$
p_{k}^{n}+q_{n-1}\left(k^{2}\right) p_{k}^{n-1}+\ldots+q_{0}\left(k^{2}\right)=0 .
$$

Пусть в системе происходят взаимодействия лишь нулевого, первого и второго порядков [5]. Каждый из информационных ресурсов $U_{i}$ может диффундировать вдоль органа управления с коэффициентом диффузии $D_{i}$. Система кинетических уравнений будет иметь вид:

$$
\begin{aligned}
\frac{\partial U_{i}}{\partial t}=a_{i}+\sum_{j=1}^{n} b_{i j} U_{j} & +\sum_{k, l=1}^{n} c_{i k l} U_{k} U_{l}+ \\
& +D_{i} \frac{\partial^{2} U_{i}}{\partial x^{2}},(i=1,2, \ldots, n) .
\end{aligned}
$$

Правая часть каждого уравнения является суммарной скоростью изменения концентрации $U_{i}$ в точке органа управления с координатой $x$. Эта скорость слагается из скоростей отдельных взаимодействий и из скорости изменения концентрации за счет диффузии. Уравнения могут быть дополнены членами вида $V_{i} \frac{\partial U_{i}}{\partial x}$, которые описывают скорость изменения концентраций за счет перемещения ресурсов $U_{i}$ вдоль органа управления с некоторой средней скоростью $V_{i}$.
Коэффициенты $a_{i^{\prime}} b_{i j} c_{i k l}, D_{i^{\prime}} V_{i}$ могут быть функциями координаты $x$.

Даже точечная система при $n>2$ трудна для решения и анализа полученных результатов. Это тем более относится к распределенным системам. Можно выполнить исследование распределенных систем, в которых участвуют лишь два вида информационных ресурсов, или две кинетические переменные $U_{1}$ и $U_{2}$. В этом случае можно записать систему уравнений, не оговаривая специально, что в системе идут лишь взаимодействия нулевого, первого и второго порядков. А именно, рассматривается система

$$
\begin{aligned}
& \frac{\partial U_{1}}{\partial t}=Q_{1}\left(U_{1}, U_{2}, x\right)+D_{1} \frac{\partial^{2} U_{1}}{\partial x^{2}} \\
& \frac{\partial U_{2}}{\partial t}=Q_{2}\left(U_{1}, U_{2}, x\right)+D_{2} \frac{\partial^{2} U_{2}}{\partial x^{2}} .
\end{aligned}
$$

В настоящее время известно большое количество конкретных систем, исследование которых сводится к поискам различных решений уравнений.

В качестве первого шага в изучении систем производится анализ устойчивости пространственно однородного стационарного решения:

$$
U_{1}=\bar{U}_{1}=\text { const, } U_{2}=\bar{U}_{2}=\text { const },
$$

где $\bar{U}_{1}$ и $\bar{U}_{2}$ являются корнями алгебраической системы уравнений:

$$
Q_{1}\left(U_{1}, U_{2}\right)=0, Q_{2}\left(U_{1}, U_{2}\right)=0
$$

и, следовательно, особыми точками соответствующей точечной системы. Так же как и в точечной системе, прежде всего необходимо исследовать решение на устойчивость. Такое исследование осуществляется с учетом конкретных краевых условий.

Исследование устойчивости пространственно однородного решения проводится по отношению к малым отклонениям от него. В большинстве случаев исследование устойчивости можно провести на основе анализа линеаризованной системы уравнений.

Пусть $U_{1}^{\prime}(t, x)$ и $U_{2}^{\prime}(t, x)$ - малые отклонения от пространственно однородных решений $U_{1}$ и $U_{2}$. Тогда для $U_{1}^{\prime}$ и $U_{2}^{\prime}$ можно записать следующую распределенную линеаризованную систему:

$$
\begin{aligned}
& \frac{\partial U_{1}^{\prime}}{\partial t}=a U_{1}^{\prime}+b U_{2}^{\prime}+D_{1} \frac{\partial^{2} U_{1}^{\prime}}{\partial x^{2}} ; \\
& \frac{\partial U_{2}^{\prime}}{\partial t}=c U_{1}^{\prime}+d U_{2}^{\prime}+D_{2} \frac{\partial^{2} U_{2}^{\prime}}{\partial x^{2}} . \\
& \text { здесь } \\
& a=\frac{\partial Q_{1}\left(\bar{U}_{1}, \bar{U}_{2}\right)}{\partial U_{1}^{\prime}}, b=\frac{\partial Q_{1}\left(\bar{U}_{1}, \bar{U}_{2}\right)}{\partial U_{2}^{\prime}}, \\
& c=\frac{\partial Q_{2}\left(\bar{U}_{1}, \bar{U}_{2}\right)}{\partial U_{1}^{\prime}}, d=\frac{\partial Q_{2}\left(\bar{U}_{1}, \bar{U}_{2}\right)}{\partial U_{2}^{\prime}} .
\end{aligned}
$$


Решение системы ищется в виде:

$$
U_{1}^{\prime}(t, x)=A e^{p t} e^{i 2 \pi r / \lambda}, U_{2}^{\prime}(t, x)=B e^{p t} e^{i 2 \pi r / \lambda} .
$$

Для бесконечного одномерного пространства значения длины волны $\lambda$ непрерывно меняются от 0 до $\infty$, а в случае отрезка $\lambda$ принимает дискретные значения. Комплексная частота $p$ определяется из квадратного уравнения

$$
\left[p-a+(2 \pi / \lambda)^{2} D_{1}\right]\left[p-d+(2 \pi / \lambda)^{2} D_{2}\right]=b c .
$$

Частный случай взаимодействия двух видов информационных ресурсов, описываемый уравнением Лотки [6]:

$$
\begin{aligned}
& \frac{\partial U_{1}}{\partial t}=k_{0}-k_{1} U_{1} U_{2}+D_{1} \frac{\partial^{2} U_{1}}{\partial x^{2}} \\
& \frac{\partial U_{2}}{\partial t}=k_{1} U_{1} U_{2}-k_{2} U_{2}+D_{2} \frac{\partial^{2} U_{2}}{\partial x^{2}}
\end{aligned}
$$

где $D_{1}$ и $D_{2}$ - соответствующие коэффициенты диффузии для информационных ресурсов с концентрациями $U_{1}$ и $U_{2}$.

Стационарные решения:

$$
\bar{U}_{1}=\frac{k_{2}}{k_{1}}, \bar{U}_{2}=\frac{k_{0}}{k_{2}} \text {. }
$$

Малые отклонения от стационарных концентраций:

$$
U_{1}^{\prime}=U_{1}(x, t)-\frac{k_{2}}{k_{1}}, U_{2}^{\prime}=U_{2}(x, t)-\frac{k_{0}}{k_{2}} \text {. }
$$

Линеаризованная система уравнений имеет следующий вид:

$$
\begin{aligned}
& \frac{\partial U_{1}^{\prime}}{\partial t}=-\frac{k_{1} k_{0}}{k_{2}} U_{1}^{\prime}-k_{2} U_{2}^{\prime}+D_{1} \frac{\partial^{2} U_{1}^{\prime}}{\partial x^{2}} ; \\
& \frac{\partial U_{2}^{\prime}}{\partial t}=k_{0} U_{1}^{\prime}+k_{2} U_{2}^{\prime} .
\end{aligned}
$$

Для того чтобы поведение этой системы имело колебательный характер, необходимо выполнение неравенства $4 k_{2}^{2} \gg k_{1} k_{0}$.

Отсутствие взаимодействия, информационный поток одного типа распространяется за счет диффузии в одномерном пространстве органа управления, в каждой точке происходит генерация данных по логистическому закону. Система такого рода описывается уравнением Фишера [7]:

$$
\frac{\partial U}{\partial t}=k U(1-U)+D \frac{\partial U^{2}}{\partial t^{2}},
$$

где скалярная функция $U(x, t)$ удовлетворяет заданным начальным и граничным условиям, а $k$ и $D$ - положительные постоянные.

Решение представляется в виде бегущей волны:

$U(x, t)=f(z), z=x+c t$,

где $c$ - скорость волны.

После подстановки в исходное уравнение функция $f(z)$ удовлетворяет уравнению

$$
D \frac{d^{2} f}{d z^{2}}-c \frac{d f}{d z}+k f(1-f)=0 .
$$

Можно записать уравнение в форме:

$$
\frac{d f}{d z}=F, D F_{z}=c F-k f(1-f),
$$

тогда траектории в фазовой плоскости $(f, F)$ будут решениями уравнения

$$
\frac{d F}{d f}=\frac{c F-k f(1-f)}{D F},
$$

которое имеет в плоскости две особые точки: $(0,0)$ и $(1,0)$. Волновое решение уравнения соответствует траектории из $(0,0)$ в $(1,0)$, остающейся в полосе $0 \leq f \leq 1$ с производной $F \geq 0$.

Вблизи точки $(0,0)$ уравнение можно линеаризовать, что дает

$$
\frac{d F}{d f} \approx \frac{c F-k f}{D F}
$$

откуда $(0,0)$ представляет собой неустойчивый узел, если $c \geq c_{\min }=2 \sqrt{k D}$. Если $0<c<c_{\min }$, особая точка $(0,0)$ является неустойчивым фокусом, в то время как при $c=0$ это центр. В этих случаях решений $f$, удовлетворяющих требуемым условиям, не может быть, так как вблизи $(0,0)$ на любой траектории найдутся точки, на которых $f<0$.

Другое необходимое условие заключается в том, что особая точка $(1,0)$ седловая. Вблизи $(1,0) f \approx 1$, и линеаризованная форма здесь имеет вид:

$$
\frac{d F}{d f} \approx \frac{c F-k(f-1)}{D F},
$$

что соответствует седловой точке для всех $c \geq 0$, так как $k$ и $D$ - положительные постоянные. Для каждого $c \geq c_{\min }=2 \sqrt{k D}$ есть единственная траектория, начинающаяся в точке $f=0$ и движущаяся к точке $f=1$ в полосе $0 \leq f \leq 1$, у которой $F>0$, за исключением точек $f=0$ и $f=1$, где $F=0$. При $c=c_{\text {min }}$ узел в точке $(0,0)$ вырожденный с двумя наклонами $\left[c \pm\left(c^{2}-4 k D\right)^{\frac{1}{2}}\right] / 2 D$, сливающимися в $c_{\min } / 2 D=\sqrt{k / D}$.

\section{2 РЕШеНИЕ ДИФФУЗИОННЫХ ЗАДАЧ С ГЕНЕРАЦИЕЙ ДАННЫХ}

В качестве математической модели распространения информационных ресурсов в органе управления в работах [2, 3] были предложены параболические уравнения теплопроводности или диффузии с различными граничными и начальными условиями. Рассматривались методы их аналитического решения. Если учесть реальную ситуацию формирования новых данных в каждой точке пространства (конкретное должностное лицо в последовательной цепочке формирования вы- 


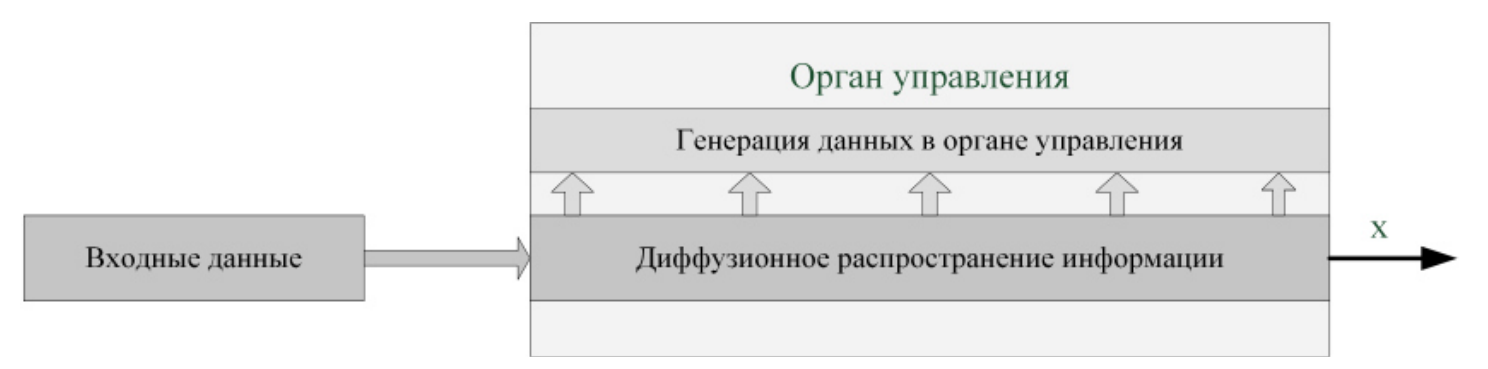

Рис. 1. Диффузионное распространение информации в органе правления с генерацией данных: $u_{t}=\alpha^{2} u_{x x}+\beta u$

ходных документов), то можно построить модель на основе диффузионного уравнения с добавлением неоднородного члена, описывающего генерацию данных, и при определенном воздействии на входе (рис. 1).

Качественное исследование нелинейной модели с логистическим законом генерации данных рассматривалась в первом разделе. Более простой экспоненциальный закон генерации позволяет получить аналитическое решение уравнения методом синус-преобразования [8].

Решаемая задача при постоянном поступлении информации на вход:

$u_{t}=\alpha^{2} u_{x x}+\beta u, 0<x<\infty, 0<t<\infty$

$u(0, t)=A$;

$u(x, 0)=0$.

Путем преобразования $u(x, t)=e^{\beta t} w(x, t)$ исходная задача приводится к простому уравнению с изменением граничного условия:

$$
\begin{aligned}
& u_{t}=\beta e^{\beta t} w+e^{\beta t} w_{t} ; \\
& u_{x x}=e^{\beta t} w_{x x} ; \\
& \beta e^{\beta t} w+e^{\beta t} w_{t}=\alpha^{2} e^{\beta t} w_{x x}+\beta e^{\beta t} w ; \\
& w_{t}=\alpha^{2} w_{x x} ; \\
& u(0, t)=e^{\beta t} w(0, t)=A, w(0, t)=A e^{-\beta t} \\
& u(x, 0)=e^{\beta \cdot 0} w(x, 0), w(x, 0)=0 .
\end{aligned}
$$

Решение задачи разбивается на три простых шага.
На первом шаге выполняется синус-преобразование Фурье по переменной $x$, тогда получается обыкновенное дифференциальное уравнение по переменной $t$ :

$$
\begin{aligned}
& F_{s}\left[w_{t}\right]=\alpha^{2} F_{s}\left[w_{x x}\right] \\
& F_{s}\left[w_{t}\right]=\frac{2}{\pi} \int_{0}^{\infty} w_{t}(x, t) \sin (\omega, x) d x= \\
&= \frac{\partial}{\partial t}\left[\frac{2}{\pi} \int_{0}^{\infty} w(x, t) \sin (\omega, x) d x\right]= \\
&=\frac{d}{d t} F_{s}[w]=\frac{d}{d t} W(t) \\
& F_{s}\left[w_{x x}\right]=\frac{2}{\pi} \omega w(0, t)-\omega^{2} F_{s}[w]= \\
&= \frac{2}{\pi} \omega w(0, t)-\omega^{2} W(t)= \\
&=\frac{2 A \omega}{\pi} e^{-\beta t}-\omega^{2} W(t) 2 A \omega \alpha^{2} \\
& \frac{d W}{d t}+\omega^{2} \alpha^{2} W(t)=\frac{2 t}{\pi} .
\end{aligned}
$$

Начальное условие для функции $W(t)$ получается применением преобразования к начальному условию $w(x, 0)=0$ :

$$
F_{s}[w(x, 0)]=W(0)=0 \text {. }
$$

На втором шаге решается полученное обыкновенное дифференциальное уравнение. Для решения можно использовать метод вариации постоянных:

$$
\begin{aligned}
& W(t)=C(t) e^{-\omega^{2} \alpha^{2} t} \\
& \frac{d C}{d t} e^{-\omega^{2} \alpha^{2} t}-C(t) \omega^{2} \alpha^{2} e^{-\omega^{2} \alpha^{2} t}+C(t) \omega^{2} \alpha^{2} e^{-\omega^{2} \alpha^{2} t}=\frac{2 A \omega \alpha^{2}}{\pi} e^{-\beta t} \\
& \frac{d C}{d t} e^{-\omega^{2} \alpha^{2} t}=\frac{2 A \omega \alpha^{2}}{\pi} e^{-\beta t} \\
& \frac{d C}{d t}=\frac{2 A \omega \alpha^{2}}{\pi} e^{\left(\omega^{2} \alpha^{2}-\beta\right) t} \\
& C(t)=\frac{2 A \omega \alpha^{2}}{\pi\left(\omega^{2} \alpha^{2}-\beta\right)} e^{\left(\omega^{2} \alpha^{2}-\beta\right) t}+C_{1}
\end{aligned}
$$




$$
\begin{aligned}
& W(0)=\left(\frac{2 A \omega \alpha^{2}}{\pi\left(\omega^{2} \alpha^{2}-\beta\right)} e^{\left(\omega^{2} \alpha^{2}-\beta\right) t}+C_{1}\right) e^{-\omega^{2} \alpha^{2} 0}=0, C_{1}=-\frac{2 A \omega \alpha^{2}}{\pi\left(\omega^{2} \alpha^{2}-\beta\right)} \\
& W(t)=\left(\frac{2 A \omega \alpha^{2}}{\pi\left(\omega^{2} \alpha^{2}-\beta\right)} e^{\left(\omega^{2} \alpha^{2}-\beta\right) t}-\frac{2 A \omega \alpha^{2}}{\pi\left(\omega^{2} \alpha^{2}-\beta\right)}\right) e^{-\omega^{2} \alpha^{2} 0}=\frac{2 A \omega \alpha^{2}}{\pi\left(\omega^{2} \alpha^{2}-\beta\right)}\left(e^{-\beta t}-e^{-\omega^{2} \alpha^{2} t}\right) .
\end{aligned}
$$

На третьем шаге применяется обратное преобразование к функции $W(t)$ :

$w(x, t)=F_{s}^{-1}[W]=F_{s}^{-1}\left[\frac{2 A \omega \alpha^{2}}{\pi\left(\omega^{2} \alpha^{2}-\beta\right)}\left(e^{-\beta t}-e^{-\omega^{2} \alpha^{2} t}\right)\right]=\int_{0}^{\infty}\left[\frac{2 A \omega \alpha^{2}}{\pi\left(\omega^{2} \alpha^{2}-\beta\right)}\left(e^{-\beta t}-e^{-\omega^{2} \alpha^{2} t}\right)\right] \sin (\omega x) d \omega$.

Решение задачи:

$u(x, t)=e^{\beta t} \int_{0}^{\infty}\left[\frac{2 A \omega \alpha^{2}}{\pi\left(\omega^{2} \alpha^{2}-\beta\right)}\left(e^{-\beta t}-e^{-\omega^{2} \alpha^{2} t}\right)\right] \sin (\omega x) d \omega$.

Задача при периодическом поступлении информации на вход:

$u_{t}=\alpha^{2} u_{x x}+\beta u, 0<x<\infty, 0<t<\infty$;

$u(0, t)=A[\cos (\sigma t)+1]$

$u(x, 0)=0$.

Путем преобразования $u(x, t)=e^{\beta t} w(x, t)$ исходная задача приводится к простому уравнению:

$$
\begin{aligned}
& u_{t}=\beta e^{\beta t} w+e^{\beta t} w_{t} ; \\
& u_{x x}=e^{\beta t} w_{x x} ; \\
& \beta e^{\beta t} w+e^{\beta t} w_{t}=\alpha^{2} e^{\beta t} w_{x x}+\beta e^{\beta t} w ; \\
& w_{t}=\alpha^{2} w_{x x} ; \\
& u(0, t)=e^{\beta t} w(0, t)=A[\cos (\sigma t)+1], \\
& \qquad w(0, t)=A[\cos (\sigma t)+1] e^{-\beta t} .
\end{aligned}
$$

Синус-преобразование Фурье по переменной $x$ с получением обыкновенного дифференциального уравнения по переменной $t$ :

$F_{s}\left[w_{t}\right]=\alpha^{2} F_{s}\left[w_{x x}\right]$

$$
\begin{gathered}
F_{s}\left[w_{t}\right]=\frac{2}{\pi} \int_{0}^{\infty} w_{t}(x, t) \sin (\omega, x) d x= \\
=\frac{\partial}{\partial t}\left[\frac{2}{\pi} \int_{0}^{\infty} w(x, t) \sin (\omega, x) d x\right]= \\
=\frac{d}{d t} F_{s}[w]=\frac{d}{d t} W(t) ;
\end{gathered}
$$$$
F_{s}\left[w_{x x}\right]=\frac{2}{\pi} \omega w(0, t)-\omega^{2} F_{s}[w]=
$$$$
=\frac{2}{\pi} \omega w(0, t)-\omega^{2} W(t)=
$$$$
=\frac{A[\cos (\sigma t)+1] \omega}{\pi} e^{-\beta t}-\omega^{2} W(t)
$$

$\frac{d W}{d t}+\omega^{2} \alpha^{2} W(t)=\frac{2 A[\cos (\sigma t)+1] \omega \alpha^{2}}{\pi} e^{-\beta t}$.

Начальное условие не изменяется:

$F_{s}[w(x, 0)]=W(0)=0$.

Решение полученного обыкновенного дифференциального уравнения методом вариации постоянных:

$W(t)=C(t) e^{-\omega^{2} \alpha^{2} t}$

$\frac{d C}{d t} e^{-\omega^{2} \alpha^{2} t}-C(t) \omega^{2} \alpha^{2} e^{-\omega^{2} \alpha^{2} t}+C(t) \omega^{2} \alpha^{2} e^{-\omega^{2} \alpha^{2} t}=\frac{A[\cos (\sigma t)+1] \omega \alpha^{2}}{\pi} e^{-\beta t}$

$\frac{d C}{d t} e^{-\omega^{2} \alpha^{2} t}=\frac{A[\cos (\sigma t)+1] \omega \alpha^{2}}{\pi} e^{-\beta t}$

$\frac{d C}{d t}=\frac{A[\cos (\sigma t)+1] \omega \alpha^{2}}{\pi} e^{\left(\omega^{2} \alpha^{2}-\beta\right) t} ;$ 


$$
\begin{aligned}
& C(t)= \frac{A \omega \alpha^{2}}{\pi}\left\{\int \cos (\sigma t) e^{\left(\omega^{2} \alpha^{2}-\beta\right) t} d t+\int e^{\left(\omega^{2} \alpha^{2}-\beta\right) t} d t\right\}+C_{1}= \\
&=\frac{A \omega \alpha^{2}}{\pi}\left\{\frac{\sigma\left(\omega^{2} \alpha^{2}-\beta\right) \sin (\sigma t) e^{\left(\omega^{2} \alpha^{2}-\beta\right) t}-\cos (\sigma t) e^{\left(\omega^{2} \alpha^{2}-\beta\right) t}}{\left(\omega^{2} \alpha^{2}-\beta\right)^{3} \sigma^{2}-1}+\frac{1}{\left(\omega^{2} \alpha^{2}-\beta\right)} e^{\left(\omega^{2} \alpha^{2}-\beta\right) t}\right\}+C_{1} ; \\
& C_{1}=-\frac{A \omega \alpha^{2}}{\pi}\left\{\frac{\sigma\left(\omega^{2} \alpha^{2}-\beta\right)}{\left(\omega^{2} \alpha^{2}-\beta\right)^{3} \sigma^{2}-1}+\frac{1}{\left(\omega^{2} \alpha^{2}-\beta\right)}\right\} ; \\
& W(t)=\frac{A \omega \alpha^{2}}{\pi}\left\{\frac{\left.\sigma\left(\omega^{2} \alpha^{2}-\beta\right) \sin (\sigma t) e^{\left(\omega^{2} \alpha^{2}-\beta\right) t}-\cos (\sigma t) e^{\left(\omega^{2} \alpha^{2}-\beta\right) t}+\frac{e^{\left(\omega^{2} \alpha^{2}-\beta\right) t}}{\left(\omega^{2} \alpha^{2}-\beta\right)}\right\} e^{-\omega^{2} \alpha^{2} t}-}{\left.\pi \alpha^{2}-\beta\right)^{3} \sigma^{2}-1}-\frac{A \omega \alpha^{2}}{\pi}\left\{\frac{\sigma\left(\omega^{2} \alpha^{2}-\beta\right)}{\left(\omega^{2} \alpha^{2}-\beta\right)^{3} \sigma^{2}-1}+\frac{1}{\left(\omega^{2} \alpha^{2}-\beta\right)}\right\} e^{-\omega^{2} \alpha^{2} t} .\right.
\end{aligned}
$$

Обратное преобразование к функции $W(t)$ :

$w(x, t)=F_{s}^{-1}[W]=\int_{0}^{\infty} W(t) \sin (\omega x) d \omega$.

Решение задачи:

$u(x, t)=e^{\beta t} \times$

$\times \int_{0}^{\infty} \frac{A \omega \alpha^{2}}{\pi}\left\{\frac{\sigma\left(\omega^{2} \alpha^{2}-\beta\right) \sin (\sigma t) e^{\left(\omega^{2} \alpha^{2}-\beta\right) t}-\cos (\sigma t) e^{\left(\omega^{2} \alpha^{2}-\beta\right) t}}{\left(\omega^{2} \alpha^{2}-\beta\right)^{3} \sigma^{2}-1}+\frac{e^{\left(\omega^{2} \alpha^{2}-\beta\right) t}}{\left(\omega^{2} \alpha^{2}-\beta\right)}\right\} e^{-\omega^{2} \alpha^{2} t} \sin (\omega x) d \omega-$ $-e^{\beta t} \int_{0}^{\infty} \frac{A \omega \alpha^{2}}{\pi}\left\{\frac{\sigma\left(\omega^{2} \alpha^{2}-\beta\right)}{\left(\omega^{2} \alpha^{2}-\beta\right)^{3} \sigma^{2}-1}+\frac{1}{\left(\omega^{2} \alpha^{2}-\beta\right)}\right\} e^{-\omega^{2} \alpha^{2} t} \sin (\omega x) d \omega$.

\section{3 РЕШЕНИЕ ДИФФУЗИОННЫХ ЗАДАЧ С КОНВЕКЦИЕЙ ДАННЫХ}

При распространении информационных ресурсов в органе управления под влиянием градиента концентрации и описываемом уравнении диффузии, возможна ситуация, когда информация передается с определенной скоростью по всему пространству под влиянием других факторов. В этом случае математической моделью может быть уравнение диффузии с конвективным членом (рис. 2).

Решаемая задача при постоянном поступлении информации на вход:

$$
\begin{aligned}
& u_{t}=\alpha^{2} u_{x x}+v u_{x}, 0<x<\infty, 0<t<\infty \\
& u(0, t)=A \\
& u(x, 0)=0
\end{aligned}
$$

Путем преобразования $u(x, t)=e^{-\frac{v\left[x+\frac{v t}{2}\right]}{2 \alpha^{2}}} w(x, t)$ исходная задача приводится к простому уравнению с изменением граничного условия:

$$
\begin{aligned}
& \left(e^{\left.-\frac{v\left[x+\frac{v t}{2}\right]}{2 \alpha^{2}} w\right)_{t}}=-\frac{v^{2}}{4 \alpha^{2}} e^{-\frac{v\left[x+\frac{v t}{2}\right]}{2 \alpha^{2}} w+e^{-\frac{v\left[x+\frac{v t}{2}\right]}{2 \alpha^{2}}} w_{t}}\right. \\
& \left(e^{-\frac{v\left[x+\frac{v t}{2}\right]}{2 \alpha^{2}} w}=-\frac{v}{2 \alpha^{2}} e^{-\frac{v\left[x+\frac{v t}{2}\right]}{2 \alpha^{2}}} w+e^{-\frac{v\left[x+\frac{v t}{2}\right]}{2 \alpha^{2}}} w_{x}\right.
\end{aligned}
$$




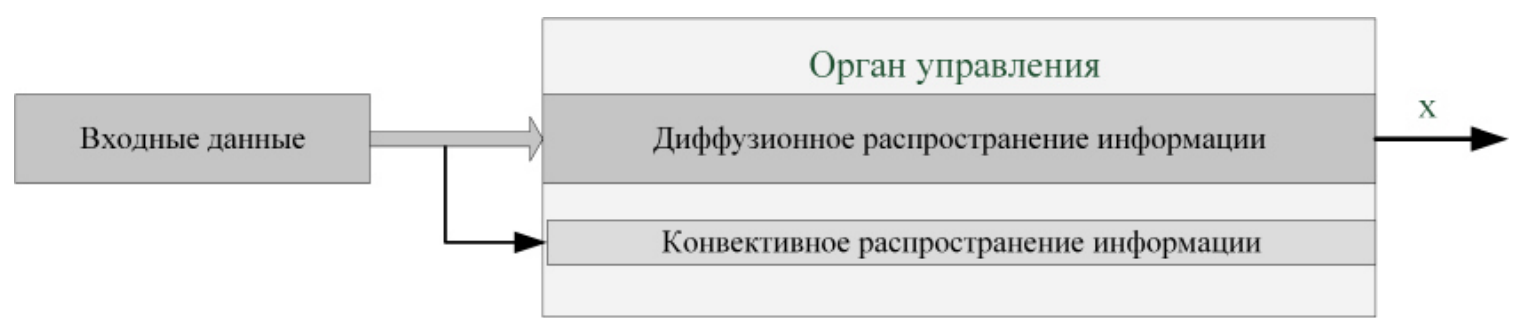

Рис. 2. Диффузионное и конвективное распространение информации в органе управления: $u_{t}=\alpha^{2} u_{x x}+v u_{x}$

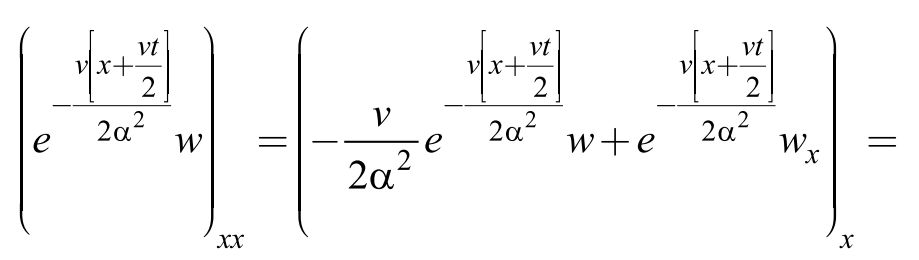

$$
\begin{aligned}
& =\frac{v^{2}}{4 \alpha^{4}} e^{-\frac{v\left[x+\frac{v t}{2}\right]}{2 \alpha^{2}}} w-\frac{v}{2 \alpha^{2}} e^{-\frac{v\left[x+\frac{v t}{2}\right]}{2 \alpha^{2}}} w_{x}-\frac{v}{2 \alpha^{2}} e^{-\frac{v\left[x+\frac{v t}{2}\right]}{2 \alpha^{2}}} w_{x}+e^{-\frac{v\left[x+\frac{v t}{2}\right]}{2 \alpha^{2}}} w_{x x} \\
& -\frac{v^{2}}{4 \alpha^{2}} e^{-\frac{v\left[x+\frac{v t}{2}\right]}{2 \alpha^{2}}} w+e^{-\frac{v\left[x+\frac{v t}{2}\right]}{2 \alpha^{2}}} w_{t}= \\
& =\alpha^{2}\left[\frac{v^{2}}{4 \alpha^{4}} e^{-\frac{v\left[x+\frac{v t}{2}\right]}{2 \alpha^{2}}} w-\frac{v}{2 \alpha^{2}} e^{-\frac{v\left[x+\frac{v t}{2}\right]}{2 \alpha^{2}}} w_{x}-\frac{v}{2 \alpha^{2}} e^{-\frac{v\left[x+\frac{v t}{2}\right]}{2 \alpha^{2}}} w_{x}+e^{-\frac{v\left[x+\frac{v t}{2}\right]}{2 \alpha^{2}}} w_{x x}\right]+ \\
& +v\left[-\frac{v}{2 \alpha^{2}} e^{-\frac{v\left[x+\frac{v t}{2}\right]}{2 \alpha^{2}}} w+e^{-\frac{v\left[x+\frac{v t}{2}\right]}{2 \alpha^{2}}} w_{x}\right]
\end{aligned}
$$

$$
\begin{aligned}
& w_{t}=\alpha^{2} w_{x x} \\
& w(0, t)=A e^{\frac{v^{2} t}{4 \alpha^{2}}} \\
& w(x, 0)=0 .
\end{aligned}
$$

Выполнение синус-преобразования Фурье по переменной $x$, с получением обыкновенного дифференциального уравнения по переменной $t$ :

$$
\begin{aligned}
& F_{s}\left[w_{t}\right]=\alpha^{2} F_{s}\left[w_{x x}\right] \\
& F_{s}\left[w_{t}\right]=\frac{2}{\pi} \int_{0}^{\infty} w_{t}(x, t) \sin (\omega, x) d x= \\
&=\frac{\partial}{\partial t}\left[\frac{2}{\pi} \int_{0}^{\infty} w(x, t) \sin (\omega, x) d x\right]= \\
&=\frac{d}{d t} F_{s}[w]=\frac{d}{d t} W(t)
\end{aligned}
$$

$$
\begin{gathered}
F_{s}\left[w_{x x}\right]=\frac{2}{\pi} \omega w(0, t)-\omega^{2} F_{s}[w]= \\
=\frac{2}{\pi} \omega w(0, t)-\omega^{2} W(t)= \\
=\frac{2 A \omega}{\pi} e^{\frac{v^{2} t}{4 \alpha^{2}}}-\omega^{2} W(t)
\end{gathered}
$$$$
\frac{d W}{d t}+\omega^{2} \alpha^{2} W(t)=\frac{2 A \omega \alpha^{2}}{\pi} e^{\frac{v^{2} t}{4 \alpha^{2}}} .
$$

Начальное условие для функции $W(t)$ получается применением преобразования к начальному условию $w(x, 0)=0$ :

$$
F_{s}[w(x, 0)]=W(0)=0 .
$$


Решение полученного обыкновенного дифференциального уравнения методом вариации постоянных:

$$
\begin{aligned}
& W(t)=C(t) e^{-\omega^{2} \alpha^{2} t} \\
& \frac{d C}{d t} e^{-\omega^{2} \alpha^{2} t}-C(t) \omega^{2} \alpha^{2} e^{-\omega^{2} \alpha^{2} t}+ \\
& \quad+C(t) \omega^{2} \alpha^{2} e^{-\omega^{2} \alpha^{2} t}=\frac{2 A \omega \alpha^{2}}{\pi} e^{\frac{v^{2} t}{4 \alpha^{2}}}
\end{aligned}
$$

$\frac{d C}{d t} e^{-\omega^{2} \alpha^{2} t}=\frac{2 A \omega \alpha^{2}}{\pi} e^{\frac{v^{2} t}{4 \alpha^{2}}}$

$\frac{d C}{d t}=\frac{2 A \omega \alpha^{2}}{\pi} e^{\left(\omega^{2} \alpha^{2}+\frac{v^{2}}{4 \alpha^{2}}\right) t} ;$

$C(t)=\frac{2 A \omega \alpha^{2}}{\pi} \frac{1}{\omega^{2} \alpha^{2}+\frac{v^{2}}{4 \alpha^{2}}} e^{\left(\omega^{2} \alpha^{2}+\frac{v^{2}}{4 \alpha^{2}}\right) t}+C_{1}$

$W(0)=\left(\frac{2 A \omega \alpha^{2}}{\pi} \frac{1}{\omega^{2} \alpha^{2}+\frac{v^{2}}{4 \alpha^{2}}} e^{\left(\omega^{2} \alpha^{2}+\frac{v^{2}}{4 \alpha^{2}}\right) 0}+C_{1}\right) e^{-\omega^{2} \alpha^{2} 0}=0$

$C_{1}=-\frac{2 A \omega \alpha^{2}}{\pi} \frac{1}{\omega^{2} \alpha^{2}+\frac{v^{2}}{4 \alpha^{2}}}$

$W(0)=\left(\frac{2 A \omega \alpha^{2}}{\pi} \frac{1}{\omega^{2} \alpha^{2}+\frac{v^{2}}{4 \alpha^{2}}} e^{\left(\omega^{2} \alpha^{2}+\frac{v^{2}}{4 \alpha^{2}}\right) t}-\frac{2 A \omega \alpha^{2}}{\pi} \frac{1}{\omega^{2} \alpha^{2}+\frac{v^{2}}{4 \alpha^{2}}}\right) e^{-\omega^{2} \alpha^{2} t}=$

$$
=\frac{2 A \omega \alpha^{2}}{\pi} \frac{1}{\omega^{2} \alpha^{2}+\frac{v^{2}}{4 \alpha^{2}}}\left(e^{\frac{v^{2}}{4 \alpha^{2}} t}-e^{-\omega^{2} \alpha^{2} t}\right) .
$$

Обратное преобразование к функции $W(t)$ :

$$
\begin{aligned}
w(x, t)=F_{s}^{-1}[W]=F_{s}^{-1}\left[\frac{2 A \omega \alpha^{2}}{\pi} \frac{1}{\omega^{2} \alpha^{2}+\frac{v^{2}}{4 \alpha^{2}}}\left(e^{\frac{v^{2}}{4 \alpha^{2}} t}-e^{-\omega^{2} \alpha^{2} t}\right]=\right. \\
=\int_{0}^{\infty} \frac{2 A \omega \alpha^{2}}{\pi} \frac{1}{\omega^{2} \alpha^{2}+\frac{v^{2}}{4 \alpha^{2}}}\left(e^{\frac{v^{2}}{4 \alpha^{2}} t}-e^{-\omega^{2} \alpha^{2} t}\right] \sin (\omega x) d \omega .
\end{aligned}
$$

\section{Решение задачи:}

$u(x, t)=e^{-\frac{v\left[x+\frac{v t}{2}\right]}{2 \alpha^{2}}} \int_{0}^{\infty}\left[\frac{2 A \omega \alpha^{2}}{\pi} \frac{1}{\omega^{2} \alpha^{2}+\frac{v^{2}}{4 \alpha^{2}}}\left(e^{\frac{v^{2}}{4 \alpha^{2}} t}-e^{-\omega^{2} \alpha^{2} t}\right)\right] \sin (\omega x) d \omega$. 
Решаемая задача при периодическом поступлении информации на вход:

$$
\begin{aligned}
& u_{t}=\alpha^{2} u_{x x}+v u_{x}, 0<x<\infty, 0<t<\infty ; \\
& u(0, t)=A[\cos (\sigma t)+1] ; \\
& u(x, 0)=0 . \\
& \text { Путем преобразования } u(x, t)=e^{-\frac{v\left[x+\frac{v t}{2}\right]}{2 \alpha^{2}}} w(x, t)
\end{aligned}
$$
исходная задача приводится к простому уравнению $\left(e^{\left.-\frac{v\left[x+\frac{v t}{2}\right]}{2 \alpha^{2}} w\right)_{t}}=-\frac{v^{2}}{4 \alpha^{2}} e^{-\frac{v\left[x+\frac{v t}{2}\right]}{2 \alpha^{2}} w+e^{-\frac{v\left[x+\frac{v t}{2}\right]}{2 \alpha^{2}}} w_{t}}\right.$
$\left(e^{-\frac{v\left[x+\frac{v t}{2}\right]}{2 \alpha^{2}}} w\right)_{x}=-\frac{v}{2 \alpha^{2}} e^{-\frac{v\left[x+\frac{v t}{2}\right]}{2 \alpha^{2}}} w+e^{-\frac{v\left[x+\frac{v t}{2}\right]}{2 \alpha^{2}}} w_{x}$ с изменением граничного условия:

$$
\begin{aligned}
\left(e^{\left.-\frac{v\left[x+\frac{v t}{2}\right]}{2 \alpha^{2}} w\right)_{x x}}=\left(-\frac{v}{2 \alpha^{2}} e^{\left.-\frac{v\left[x+\frac{v t}{2}\right]}{2 \alpha^{2}} w+e^{-\frac{v\left[x+\frac{v t}{2}\right]}{2 \alpha^{2}}} w_{x}\right)_{x}}=\right.\right. \\
=\frac{v}{4 \alpha^{4}} e^{-\frac{v\left[x+\frac{v t}{2}\right]}{2 \alpha^{2}} w-\frac{v}{2 \alpha^{2}} e^{-\frac{v\left[x+\frac{v t}{2}\right]}{2 \alpha^{2}}} w_{x}-\frac{v}{2 \alpha^{2}} e^{-\frac{v\left[x+\frac{v t}{2}\right]}{2 \alpha^{2}}} w_{x}+e^{-\frac{v\left[x+\frac{v t}{2}\right]}{2 \alpha^{2}}} w_{x x}}
\end{aligned}
$$$$
-\frac{v^{2}}{4 \alpha^{2}} e^{-\frac{v\left[x+\frac{v t}{2}\right]}{2 \alpha^{2}}} w+e^{-\frac{v\left[x+\frac{v t}{2}\right]}{2 \alpha^{2}}} w_{t}=
$$$$
=\alpha^{2}\left[\frac{v^{2}}{4 \alpha^{4}} e^{-\frac{v\left[x+\frac{v t}{2}\right]}{2 \alpha^{2}}} w-\frac{v}{2 \alpha^{2}} e^{-\frac{v\left[x+\frac{v t}{2}\right]}{2 \alpha^{2}}} w_{x}-\frac{v}{2 \alpha^{2}} e^{-\frac{v\left[x+\frac{v t}{2}\right]}{2 \alpha^{2}}} w_{x}+e^{-\frac{v\left[x+\frac{v t}{2}\right]}{2 \alpha^{2}}} w_{x x}\right]+
$$$$
+v\left[-\frac{v}{2 \alpha^{2}} e^{-\frac{v\left[x+\frac{v t}{2}\right]}{2 \alpha^{2}}} w+e^{-\frac{v\left[x+\frac{v t}{2}\right]}{2 \alpha^{2}}} w_{x}\right]
$$

$w_{t}=\alpha^{2} w_{x x}$

$$
\begin{aligned}
& w(0, t)=A[\cos (\sigma t)+1] e^{\frac{v^{2} t}{4 \alpha^{2}}} ; \\
& w(x, 0)=0 .
\end{aligned}
$$

Выполнение синус-преобразования Фурье по переменной $x$ с получением обыкновенного дифференциального уравнения по переменной $t$ :

$$
F_{s}\left[w_{t}\right]=\alpha^{2} F_{s}\left[w_{x x}\right]
$$

$$
\begin{gathered}
F_{s}\left[w_{t}\right]=\frac{2}{\pi} \int_{0}^{\infty} w_{t}(x, t) \sin (\omega, x) d x= \\
=\frac{\partial}{\partial t}\left[\frac{2}{\pi} \int_{0}^{\infty} w(x, t) \sin (\omega, x) d x\right]= \\
=\frac{d}{d t} F_{s}[w]=\frac{d}{d t} W(t)
\end{gathered}
$$




$$
\begin{aligned}
& F_{s}\left[w_{x x}\right]=\frac{2}{\pi} \omega w(0, t)-\omega^{2} F_{s}[w]=\frac{2}{\pi} \omega w(0, t)-\omega^{2} W(t)=\frac{2 A[\cos (\sigma t)+1] \omega}{\pi} e^{\frac{v^{2} t}{4 \alpha^{2}}}-\omega^{2} W(t) \\
& \frac{d W}{d t}+\omega^{2} \alpha^{2} W(t)=\frac{2 A[\cos (\sigma t)+1] \omega \alpha^{2}}{\pi} e^{\frac{v^{2} t}{4 \alpha^{2}}}
\end{aligned}
$$

Начальное условие для функции $W(t)$ получается применением преобразования к начальному условию $w(x, 0)=0$ :

$F_{s}[w(x, 0)]=W(0)=0$.

Решение полученного обыкновенного дифференциального уравнения методом вариации постоянных: $W(t)=C(t) e^{-\omega^{2} \alpha^{2} t} ;$

$\frac{d C}{d t} e^{-\omega^{2} \alpha^{2} t}-C(t) \omega^{2} \alpha^{2} e^{-\omega^{2} \alpha^{2} t}+C(t) \omega^{2} \alpha^{2} e^{-\omega^{2} \alpha^{2} t}=\frac{2 A[\cos (\sigma t)+1] \omega \alpha^{2}}{\pi} e^{\frac{v^{2} t}{4 \alpha^{2}}} ;$

$\frac{d C}{d t} e^{-\omega^{2} \alpha^{2} t}=\frac{2 A[\cos (\sigma t)+1] \omega \alpha^{2}}{\pi} e^{\frac{v^{2} t}{4 \alpha^{2}}} ;$

$\frac{d C}{d t}=\frac{2 A[\cos (\sigma t)+1] \omega \alpha^{2}}{\pi} e^{\left(\omega^{2} \alpha^{2}+\frac{v^{2}}{4 \alpha^{2}}\right) t} ;$

$C(t)=\frac{A \omega \alpha^{2}}{\pi}\left\{\int \cos (\sigma t) e^{\left(\omega^{2} \alpha^{2}+\frac{v^{2}}{4 \alpha^{2}}\right) t} d t+\int e^{\left(\omega^{2} \alpha^{2}+\frac{v^{2}}{4 \alpha^{2}}\right) t} d t\right\}+C_{1}=$

$=\frac{A \omega \alpha^{2}}{\pi}\left\{\frac{\sigma\left(\omega^{2} \alpha^{2}+\frac{v^{2}}{4 \alpha^{2}}\right) \sin (\sigma t) e^{\left(\omega^{2} \alpha^{2}+\frac{v^{2}}{4 \alpha^{2}}\right) t}-\cos (\sigma t) e^{\left(\omega^{2} \alpha^{2}+\frac{v^{2}}{4 \alpha^{2}}\right) t}}{\left(\omega^{2} \alpha^{2}+\frac{v^{2}}{4 \alpha^{2}}\right)^{3} \sigma^{2}-1}+\frac{1}{\left(\omega^{2} \alpha^{2}+\frac{v^{2}}{4 \alpha^{2}}\right)} e^{\left(\omega^{2} \alpha^{2}+\frac{v^{2}}{4 \alpha^{2}}\right) t}\right\}+C_{1} ;$

$C_{1}=-\frac{A \omega \alpha^{2}}{\pi}\left\{\frac{\sigma\left(\omega^{2} \alpha^{2}+\frac{v^{2}}{4 \alpha^{2}}\right)}{\left(\omega^{2} \alpha^{2}+\frac{v^{2}}{4 \alpha^{2}}\right)^{3} \sigma^{2}-1}+\frac{1}{\left(\omega^{2} \alpha^{2}+\frac{v^{2}}{4 \alpha^{2}}\right)}\right\}$

$W(t)=\frac{A \omega \alpha^{2} e^{-\omega^{2} \alpha^{2} t}}{\pi}\left\{\frac{\sigma\left(\omega^{2} \alpha^{2}+\frac{v^{2}}{4 \alpha^{2}}\right) \sin (\sigma t) e^{\left(\omega^{2} \alpha^{2}+\frac{v^{2}}{4 \alpha^{2}}\right) t}-\cos (\sigma t) e^{\left(\omega^{2} \alpha^{2}+\frac{v^{2}}{4 \alpha^{2}}\right) t}}{\left(\omega^{2} \alpha^{2}+\frac{v^{2}}{4 \alpha^{2}}\right)^{3} \sigma^{2}-1}+\right.$

$$
\left.+\frac{1}{\left(\omega^{2} \alpha^{2}+\frac{v^{2}}{4 \alpha^{2}}\right)} e^{\left(\omega^{2} \alpha^{2}+\frac{v^{2}}{4 \alpha^{2}}\right)}\right\}-\frac{A \omega \alpha^{2} e^{-\omega^{2} \alpha^{2} t}}{\pi}\left\{\frac{\sigma\left(\omega^{2} \alpha^{2}+\frac{v^{2}}{4 \alpha^{2}}\right)}{\left(\omega^{2} \alpha^{2}+\frac{v^{2}}{4 \alpha^{2}}\right)^{3} \sigma^{2}-1}+\frac{1}{\left(\omega^{2} \alpha^{2}+\frac{v^{2}}{4 \alpha^{2}}\right)}\right\} .
$$


Применение обратного преобразования к функции $W(t)$ :

$w(x, t)=F_{s}^{-1}[W]=\int_{0}^{\infty} W(t) \sin (\omega x) d \omega$.

Решение задачи:

$u(x, t)=e^{-\frac{v\left[x+\frac{v t}{2}\right]}{2 \alpha^{2}}} \frac{A \omega \alpha^{2} e^{-\omega^{2} \alpha^{2} t}}{\pi} \times$

$\times \int_{0}^{\infty}\left\{\frac{\sigma\left(\omega^{2} \alpha^{2}+\frac{v^{2}}{4 \alpha^{2}}\right) \sin (\sigma t) e^{\left(\omega^{2} \alpha^{2}+\frac{v^{2}}{4 \alpha^{2}}\right) t}-\cos (\sigma t) e^{\left(\omega^{2} \alpha^{2}+\frac{v^{2}}{4 \alpha^{2}}\right) t}}{\left(\omega^{2} \alpha^{2}+\frac{v^{2}}{4 \alpha^{2}}\right)^{3} \sigma^{2}-1}+\right.$

$+\frac{1}{\left(\omega^{2} \alpha^{2}+\frac{v^{2}}{4 \alpha^{2}}\right)} e^{\left(\omega^{2} \alpha^{2}+\frac{v^{2}}{4 \alpha^{2}}\right)} t \sin (\omega x) d \omega-$

$-e^{-\frac{v\left[x+\frac{v t}{2}\right]}{2 \alpha^{2}}} \frac{2 \omega \alpha^{2} e^{-\omega^{2} \alpha^{2} t}}{\pi} \int_{0}^{\infty}\left\{\frac{\sigma\left(\omega^{2} \alpha^{2}+\frac{v^{2}}{4 \alpha^{2}}\right)}{\left(\omega^{2} \alpha^{2}+\frac{v^{2}}{4 \alpha^{2}}\right)^{3} \sigma^{2}-1}+\frac{1}{\left(\omega^{2} \alpha^{2}+\frac{v^{2}}{4 \alpha^{2}}\right)}\right\} \sin (\omega x) d \omega$.

\section{4 РЕШЕНИЕ ДИФФУЗИОННЫХ ЗАДАЧ С ГЕНЕРАЦИЕЙ} И КОНВЕКЦИЕЙ ДАННЫХ

Учет диффузионного и конвективного распространения информации в органе управления, а также генерации данных в каждой точке пространства позволяет получить решение после двух этапов преобразования переменных (рис. 3).

Решаемая задача при постоянном поступлении информации на вход:

$u_{t}=\alpha^{2} u_{x x}+v u_{x}+\beta u, 0<x<\infty, 0<t<\infty ;$

$$
\begin{aligned}
& u(0, t)=A \\
& u(x, 0)=0
\end{aligned}
$$

Путем двух последовательных преобразований исходная задача приводится к простому уравнению:

$$
\begin{aligned}
& u(x, t)=e^{-\frac{v\left[x+\frac{v t}{2}\right]}{2 \alpha^{2}} w(x, t) ;} \\
& w(x, t)=e^{\beta t} s(x, t) ; \\
& u(x, t)=e^{-\frac{v\left[x+\frac{v t}{2}\right]}{2 \alpha^{2}}+\beta t} s(x, t) ;
\end{aligned}
$$

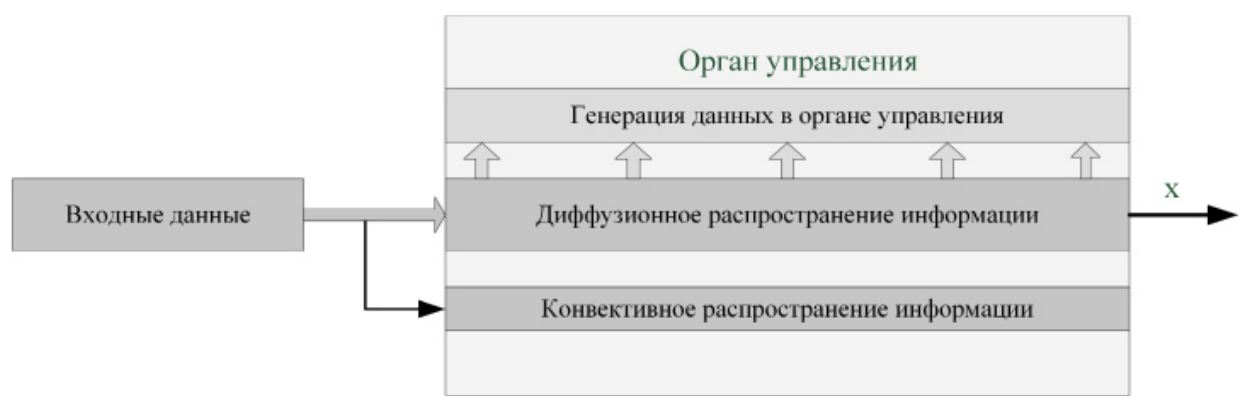

Рис. 3. Диффузионное и конвективное распространение информации в органе управления с генерацией данных: $u_{t}=\alpha^{2} u_{x x}+v u_{x}+\beta u$ 


$$
\begin{aligned}
& -\frac{v^{2}}{4 \alpha^{2}} e^{-\frac{v\left[x+\frac{v t}{2}\right]}{2 \alpha^{2}}} w+e^{-\frac{v\left[x+\frac{v t}{2}\right]}{2 \alpha^{2}}} w_{t}= \\
& =\alpha^{2}\left[\frac{v^{2}}{4 \alpha^{4}} e^{-\frac{v\left[x+\frac{v t}{2}\right]}{2 \alpha^{2}}} w-\frac{v}{2 \alpha^{2}} e^{-\frac{v\left[x+\frac{v t}{2}\right]}{2 \alpha^{2}}} w_{x}-\frac{v}{2 \alpha^{2}} e^{-\frac{v\left[x+\frac{v t}{2}\right]}{2 \alpha^{2}}} w_{x}+e^{-\frac{v\left[x+\frac{v t}{2}\right]}{2 \alpha^{2}}} w_{x x}\right]+ \\
& +v\left[-\frac{v}{2 \alpha^{2}} e^{-\frac{v\left[x+\frac{v t}{2}\right]}{2 \alpha^{2}}} w+e^{-\frac{\left[x+\frac{v t}{2}\right]}{2 \alpha^{2}}} w_{x}\right]+\beta\left(e^{-\frac{v\left[x+\frac{v t}{2}\right]}{2 \alpha^{2}}} w(x, t)\right)
\end{aligned}
$$

$w_{t}=\alpha^{2} w_{x x}+\beta w$

$\beta e^{\beta t} z+e^{\beta t} z_{t}=\alpha^{2} e^{\beta t} z_{x x}+\beta e^{\beta t} z$;

$z_{t}=\alpha^{2} z_{x x}$

$z(0, t)=A e^{\left(\frac{v^{2}}{4 \alpha^{2}}-\beta\right) t} ;$
$z(x, 0)=0$.

Выполнение синус-преобразования Фурье по переменной $x$ с получением обыкновенного дифференциального уравнения по переменной $t$ :

$$
\begin{aligned}
& F_{s}\left[z_{t}\right]=\alpha^{2} F_{s}\left[z_{x x}\right] \\
& F_{s}\left[z_{t}\right]=\frac{2}{\pi} \int_{0}^{\infty} z_{t}(x, t) \sin (\omega, x) d x= \\
&= \frac{\partial}{\partial t}\left[\frac{2}{\pi} \int_{0}^{\infty} w z(x, t) \sin (\omega, x) d x\right]= \\
&=\frac{d}{d t} F_{s}[z]=\frac{d}{d t} Z(t) ; \\
& F_{s}\left[z_{x x}\right]=\frac{2}{\pi} \omega z(0, t)-\omega^{2} F_{s}[z]= \\
&=\frac{2}{\pi} \omega z(0, t)-\omega^{2} Z(t)= \\
&=\frac{2 A \omega}{\pi} e^{\left(\frac{v^{2}}{4 \alpha^{2}}-\beta\right) t}-\omega^{2} Z(t)
\end{aligned}
$$$$
\frac{d Z}{d t}+\omega^{2} \alpha^{2} Z(t)=\frac{2 A \omega \alpha^{2}}{\pi} e^{\left(\frac{v^{2}}{4 \alpha^{2}}-\beta\right) t} .
$$

Начальное условие для функции $Z(t)$ получается применением преобразования к начальному условию $z(x, 0)=0$ :

$$
F_{s}[z(x, 0)]=Z(0)=0 .
$$

Решение полученного обыкновенного дифференциального уравнения методом вариации постоянных:

$$
Z(t)=C(t) e^{-\omega^{2} \alpha^{2} t}
$$

$$
\frac{d C}{d t} e^{-\omega^{2} \alpha^{2} t}-C(t) \omega^{2} \alpha^{2} e^{-\omega^{2} \alpha^{2} t}+
$$

$$
\begin{gathered}
+C(t) \omega^{2} \alpha^{2} e^{-\omega^{2} \alpha^{2} t}=\frac{2 A \omega \alpha^{2}}{\pi} e^{\left(\frac{v^{2}}{4 \alpha^{2}}-\beta\right) t} \\
\frac{d C}{d t} e^{-\omega^{2} \alpha^{2} t}=\frac{2 A \omega \alpha^{2}}{\pi} e^{\left(\frac{v^{2}}{4 \alpha^{2}}-\beta\right) t} ; \\
\frac{d C}{d t}=\frac{2 A \omega \alpha^{2}}{\pi} e^{\left(\omega^{2} \alpha^{2}+\frac{v^{2}}{4 \alpha^{2}}-\beta\right) t} ; \\
C(t)=\frac{2 A \omega \alpha^{2}}{\pi} \frac{1}{\omega^{2} \alpha^{2}+\frac{v^{2}}{4 \alpha^{2}}} e^{\left(\omega^{2} \alpha^{2}+\frac{v^{2}}{4 \alpha^{2}}-\beta\right) t}+C_{1} ;
\end{gathered}
$$

$Z(0)=\left(\frac{2 A \omega \alpha^{2}}{\pi} \frac{1}{\omega^{2} \alpha^{2}+\frac{v^{2}}{4 \alpha^{2}}} e^{\left(\omega^{2} \alpha^{2}+\frac{v^{2}}{4 \alpha^{2}}-\beta\right) 0}+C_{1}\right) e^{-\omega^{2} \alpha^{2} 0}=0$ 


$$
\begin{aligned}
& C_{1}=-\frac{2 A \omega \alpha^{2}}{\pi} \frac{1}{\omega^{2} \alpha^{2}+\frac{v^{2}}{4 \alpha^{2}}-\beta} ; \\
& Z(t)=\left(\frac{2 A \omega \alpha^{2}}{\pi} \frac{1}{\omega^{2} \alpha^{2}+\frac{v^{2}}{4 \alpha^{2}}-\beta} e^{\left(\omega^{2} \alpha^{2}+\frac{v^{2}}{4 \alpha^{2}}-\beta\right) t}-\frac{2 A \omega \alpha^{2}}{\pi} \frac{1}{\omega^{2} \alpha^{2}+\frac{v^{2}}{4 \alpha^{2}}-\beta}\right) e^{-\omega^{2} \alpha^{2} t}= \\
& =\frac{2 A \omega \alpha^{2}}{\pi} \frac{1}{\omega^{2} \alpha^{2}+\frac{v^{2}}{4 \alpha^{2}}-\beta}\left(e^{\left(\frac{v^{2}}{4 \alpha^{2}}-\beta\right) t}-e^{-\omega^{2} \alpha^{2} t}\right) \text {. }
\end{aligned}
$$

Обратное преобразование к функции $Z(t)$ :

$$
\begin{aligned}
& z(x, t)=F_{s}^{-1}[Z]=F_{s}^{-1}\left[\frac{2 A \omega \alpha^{2}}{\pi} \frac{1}{\omega^{2} \alpha^{2}+\frac{v^{2}}{4 \alpha^{2}}-\beta}\left(e^{\left(\frac{v^{2}}{4 \alpha^{2}}-\beta\right) t}-e^{-\omega^{2} \alpha^{2} t}\right)\right]= \\
& =\int_{0}^{\infty}\left[\frac{2 A \omega \alpha^{2}}{\pi} \frac{1}{\omega^{2} \alpha^{2}+\frac{v^{2}}{4 \alpha^{2}}-\beta}\left(e^{\left(\frac{v^{2}}{4 \alpha^{2}}-\beta\right) t}-e^{-\omega^{2} \alpha^{2} t}\right)\right] \sin (\omega x) d \omega .
\end{aligned}
$$

Решение задачи:

$u(x, t)=e^{-\frac{v\left[x+\frac{v t}{2}\right]}{2 \alpha^{2}}+\beta t} \int_{0}^{\infty}\left[\frac{2 A \omega \alpha^{2}}{\pi} \frac{1}{\omega^{2} \alpha^{2}+\frac{v^{2}}{4 \alpha^{2}}-\beta}\left(e^{\left(\frac{v^{2}}{4 \alpha^{2}}-\beta\right) t}-e^{-\omega^{2} \alpha^{2} t}\right] \sin (\omega x) d \omega\right.$.

Решаемая задача с периодическим поступлением информации на вход:

$u_{t}=\alpha^{2} u_{x x}+v u_{x}+\beta u, 0<x<\infty, 0<t<\infty$;

$u(0, t)=A[\cos (\sigma t)+1]$

$u(x, 0)=0$.

Путем двух последовательных преобразований исходная задача приводится к простому уравнению:

$$
\begin{aligned}
& u(x, t)=e^{-\frac{v\left[x+\frac{v t}{2}\right]}{2 \alpha^{2}} w(x, t) ;} \\
& w(x, t)=e^{\beta t} z(x, t) ; \\
& u(x, t)=e^{-\frac{v\left[x+\frac{v t}{2}\right]}{2 \alpha^{2}}+\beta t} z(x, t) ;
\end{aligned}
$$




$$
\begin{aligned}
& -\frac{v^{2}}{4 \alpha^{2}} e^{-\frac{v\left[x+\frac{v t}{2}\right]}{2 \alpha^{2}} w+e^{-\frac{v\left[x+\frac{v t}{2}\right]}{2 \alpha^{2}}} w_{t}}= \\
& =\alpha^{2}\left[\frac{v^{2}}{4 \alpha^{4}} e^{-\frac{v\left[x+\frac{v t}{2}\right]}{2 \alpha^{2}}} w-\frac{v}{2 \alpha^{2}} e^{-\frac{v\left[x+\frac{v t}{2}\right]}{2 \alpha^{2}}} w_{x}-\frac{v}{2 \alpha^{2}} e^{-\frac{v\left[x+\frac{v t}{2}\right]}{2 \alpha^{2}}} w_{x}+e^{-\frac{v\left[x+\frac{v t}{2}\right]}{2 \alpha^{2}}} w_{x x}\right]+ \\
& +v\left[-\frac{v}{2 \alpha^{2}} e^{-\frac{v\left[x+\frac{v t}{2}\right]}{2 \alpha^{2}} w+e^{-\left[x+\frac{v t}{2}\right]}} w_{x}\right]+\beta\left[e^{-\frac{v\left[x+\frac{v t}{2}\right]}{2 \alpha^{2}} w(x, t)} w ;\right.
\end{aligned}
$$

$$
\begin{aligned}
& w_{t}=\alpha^{2} w_{x x}+\beta w \\
& \beta e^{\beta t} s+e^{\beta t} s_{t}=\alpha^{2} e^{\beta t} s_{x x}+\beta e^{\beta t} s \\
& z_{t}=\alpha^{2} z_{x x} ; \\
& z(0, t)=A[\cos (\sigma t)+1] e^{\left(\frac{v^{2}}{4 \alpha^{2}}-\beta\right) t} \\
& z(x, 0)=0 .
\end{aligned}
$$

Выполнение синус-преобразования Фурье по переменной $x$ с получением обыкновенного дифференциального уравнения по переменной $t$ :

$$
\begin{aligned}
& F_{s}\left[z_{t}\right]=\alpha^{2} F_{s}\left[z_{x x}\right] ; \\
& F_{s}\left[z_{t}\right]= \frac{2}{\pi} \int_{0}^{\infty} z_{t}(x, t) \sin (\omega, x) d x= \\
&=\frac{\partial}{\partial t}\left[\frac{2}{\pi} \int_{0}^{\infty} z(x, t) \sin (\omega, x) d x\right]= \\
&=\frac{d}{d t} F_{s}[z]=\frac{d}{d t} Z(t) ;
\end{aligned}
$$

$$
\begin{aligned}
F_{s}\left[z_{x x}\right] & =\frac{2}{\pi} \omega z(0, t)-\omega^{2} F_{s}[z]= \\
= & \frac{2}{\pi} \omega z(0, t)-\omega^{2} Z(t)= \\
& =\frac{2 A[\cos (\sigma t)+1] \omega}{\pi} e^{\frac{v^{2} t}{4 \alpha^{2}}}-\omega^{2} Z(t)
\end{aligned}
$$

$\frac{d Z}{d t}+\omega^{2} \alpha^{2} Z(t)=\frac{2 A[\cos (\sigma t)+1] \omega \alpha^{2}}{\pi} e^{\left(\frac{v^{2}}{4 \alpha^{2}}-\beta\right) t}$

Начальное условие для функции $Z(t)$ получается применением преобразования к начальному условию $z(x, 0)=0$ :

$$
F_{s}[z(x, 0)]=Z(0)=0 \text {. }
$$

Решение полученного обыкновенного дифференциального уравнения методом вариации постоянных:

$$
Z(t)=C(t) e^{-\omega^{2} \alpha^{2} t}
$$

$$
\begin{aligned}
& \frac{d C}{d t} e^{-\omega^{2} \alpha^{2} t}-C(t) \omega^{2} \alpha^{2} e^{-\omega^{2} \alpha^{2} t}+C(t) \omega^{2} \alpha^{2} e^{-\omega^{2} \alpha^{2} t}=\frac{2 A[\cos (\sigma t)+1] \omega \alpha^{2}}{\pi} e^{\left(\frac{v^{2}}{4 \alpha^{2}}-\beta\right) t} \\
& \frac{d C}{d t} e^{-\omega^{2} \alpha^{2} t}=\frac{2 A[\cos (\sigma t)+1] \omega \alpha^{2}}{\pi} e^{\left(\frac{v^{2}}{4 \alpha^{2}}-\beta\right) t} ; \\
& \frac{d C}{d t}=\frac{2 A[\cos (\sigma t)+1] \omega \alpha^{2}}{\pi} e^{\left(\omega^{2} \alpha^{2}+\frac{v^{2}}{4 \alpha^{2}}-\beta\right) t}
\end{aligned}
$$




$$
\begin{aligned}
& C(t)=\frac{A \omega \alpha^{2}}{\pi}\left\{\int \cos (\sigma t) e^{\left(\omega^{2} \alpha^{2}+\frac{v^{2}}{4 \alpha^{2}}-\beta\right) t} d t+\int e^{\left(\omega^{2} \alpha^{2}+\frac{v^{2}}{4 \alpha^{2}}-\beta\right) t} d t\right\}+C_{1}= \\
& =\frac{A \omega \alpha^{2}}{\pi}\left\{\frac{\sigma\left(\omega^{2} \alpha^{2}+\frac{v^{2}}{4 \alpha^{2}}-\beta\right) \sin (\sigma t) e^{\left(\omega^{2} \alpha^{2}+\frac{v^{2}}{4 \alpha^{2}}-\beta\right) t}-\cos (\sigma t) e^{\left(\omega^{2} \alpha^{2}+\frac{v^{2}}{4 \alpha^{2}}-\beta\right) t}}{\left(\omega^{2} \alpha^{2}+\frac{v^{2}}{4 \alpha^{2}}-\beta\right)^{3} \sigma^{2}-1}+\right. \\
& \left.+\frac{1}{\left(\omega^{2} \alpha^{2}+\frac{v^{2}}{4 \alpha^{2}}-\beta\right)} e^{\left(\omega^{2} \alpha^{2}+\frac{v^{2}}{4 \alpha^{2}}-\beta\right) t}\right\}+C_{1} \\
& C_{1}=-\frac{A \omega \alpha^{2}}{\pi}\left\{\frac{\sigma\left(\omega^{2} \alpha^{2}+\frac{v^{2}}{4 \alpha^{2}}-\beta\right)}{\left(\omega^{2} \alpha^{2}+\frac{v^{2}}{4 \alpha^{2}}-\beta\right)^{3} \sigma^{2}-1}+\frac{1}{\left(\omega^{2} \alpha^{2}+\frac{v^{2}}{4 \alpha^{2}}-\beta\right)}\right\} \\
& Z(t)=\frac{A \omega \alpha^{2} e^{-\omega^{2} \alpha^{2} t}}{\pi}\left\{\frac{\sigma\left(\omega^{2} \alpha^{2}+\frac{v^{2}}{4 \alpha^{2}}-\beta\right) \sin (\sigma t) e^{\left(\omega^{2} \alpha^{2}+\frac{v^{2}}{4 \alpha^{2}}-\beta\right) t}-\cos (\sigma t) e^{\left(\omega^{2} \alpha^{2}+\frac{v^{2}}{4 \alpha^{2}}-\beta\right) t}}{\left(\omega^{2} \alpha^{2}+\frac{v^{2}}{4 \alpha^{2}}-\beta\right)^{3} \sigma^{2}-1}+\right. \\
& \left.+\frac{1}{\left(\omega^{2} \alpha^{2}+\frac{v^{2}}{4 \alpha^{2}}-\beta\right)} e^{\left(\omega^{2} \alpha^{2}+\frac{v^{2}}{4 \alpha^{2}}-\beta\right) t}\right\}-\frac{A \omega \alpha^{2} e^{-\omega^{2} \alpha^{2} t}}{\pi}\left\{\frac{\sigma\left(\omega^{2} \alpha^{2}+\frac{v^{2}}{4 \alpha^{2}}-\beta\right)}{\left(\omega^{2} \alpha^{2}+\frac{v^{2}}{4 \alpha^{2}}-\beta\right)^{3} \sigma^{2}-1}+\frac{1}{\left(\omega^{2} \alpha^{2}+\frac{v^{2}}{4 \alpha^{2}}-\beta\right)}\right\} .
\end{aligned}
$$

Обратное преобразование к функции $Z(t)$ :

$z(x, t)=F_{s}^{-1}[Z]=\int_{0}^{\infty} Z(t) \sin (\omega x) d \omega$. 


$$
\begin{aligned}
& \begin{array}{l}
\text { Решение задачи: } \\
u(x, t)=e^{-\frac{v\left[x+\frac{v t}{2}\right]}{2 \alpha^{2}}+\beta t} \frac{A \omega \alpha^{2} e^{-\omega^{2} \alpha^{2} t}}{\pi} \times
\end{array} \\
& \times \int_{0}^{\infty} \frac{\sigma\left(\omega^{2} \alpha^{2}+\frac{v^{2}}{4 \alpha^{2}}-\beta\right) \sin (\sigma t) e^{\left(\omega^{2} \alpha^{2}+\frac{v^{2}}{4 \alpha^{2}}-\beta\right) t}-\cos (\sigma t) e^{\left(\omega^{2} \alpha^{2}+\frac{v^{2}}{4 \alpha^{2}}-\beta\right) t}}{\left(\omega^{2} \alpha^{2}+\frac{v^{2}}{4 \alpha^{2}}-\beta\right)^{3} \sigma^{2}-1}+ \\
& \left.+\frac{1}{\left(\omega^{2} \alpha^{2}+\frac{v^{2}}{4 \alpha^{2}}-\beta\right)} e^{\left(\omega^{2} \alpha^{2}+\frac{v^{2}}{4 \alpha^{2}}-\beta\right) t}\right\} \sin (\omega x) d \omega- \\
& -e^{-\frac{v\left[x+\frac{v t}{2}\right]}{2 \alpha^{2}}+\beta t} \frac{A \omega \alpha^{2} e^{-\omega^{2} \alpha^{2} t}}{\pi} \int_{0}^{\infty}\left\{\frac{\sigma\left(\omega^{2} \alpha^{2}+\frac{v^{2}}{4 \alpha^{2}}-\beta\right)}{\left(\omega^{2} \alpha^{2}+\frac{v^{2}}{4 \alpha^{2}}-\beta\right)^{3} \sigma^{2}-1}+\frac{1}{\left(\omega^{2} \alpha^{2}+\frac{v^{2}}{4 \alpha^{2}}-\beta\right)}\right\} \sin (\omega x) d \omega .
\end{aligned}
$$

На рисунке 4 представлены примерные графики распространения информации при постоянном и периодическом поступлении информации на вход органа управления.

\section{ЗАКЛЮЧЕНИЕ}

В настоящее время, несмотря на высокую оснащенность компьютерной техникой и современными информационными технологиями органов управления, существенного повышения качества управления не достигнуто. Возможности средств автоматизации содержательной переработки информации используются не в полной мере. Более того там, где они могут

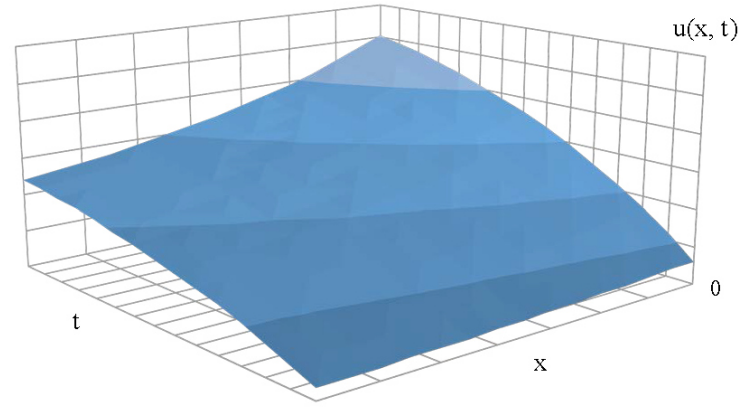

принести наибольший эффект, при обеспечении принятия своевременных и обоснованных решений на всех уровнях управления, они практически не используются. Причиной этого является недостаточная изученность и формализация сложных законов управления в обществе. Кроме того, работа в этом направлении ведется бессистемно, выделяются недостаточные ресурсы, создаваемые средства содержательной обработки информации не материализуются в форме, позволяющей их накапливание, распространение, совершенствование, а также эффективное использование [12].

Выбор дифференциальных уравнений для моделирования информационных процессов органов

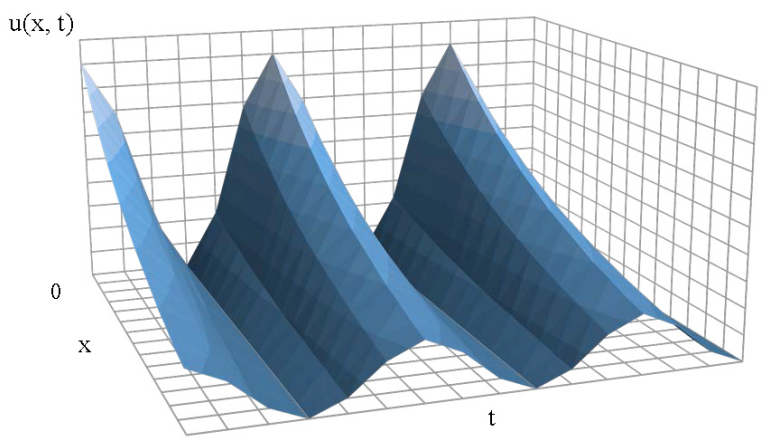

Рис. 4. Примерные графики распространения информации в органе управления при разных видах воздействия 
управления обусловлен двумя причинами. Первая причина заключается в эффективном применении дифференциальных уравнений в качестве средств решения прикладных задач естествознания и техники. Вторая причина - аналогия информационных процессов с функционированием различных систем, где успешно применяется данный математический аппарат. Приведенные в статье результаты подтверждают перспективность таких исследований, но не исключают возможность других подходов, в том числе на основе специальных теорий, созданных для описания трудноформализуемой творческой деятельности должностных лиц органов управления.

\section{СПИСОК ЛИТЕРАТУРЫ}

1. Иванов А.К. Разработка и применение математических моделей иерархических АСУ. - Ульяновск : УлГТУ, 2017. - 262 с.

2. Иванов А.К., Береснев Ю.И. Динамика распределения информации в иерархических системах // Автоматизация процессов управления. - 2017. - № 4 (50). C. 25-35.

3. Иванов А.К. Математические модели информационного пространства иерархических систем // Автоматизация процессов управления. - 2018. - № 1 (51). С. 47-57.

4. Алексеев В.В. Физическое и математическое моделирование экосистем. - СПб : Гидрометеоиздат, 1992. - 367 c.

5. Романовский Ю.М., Степанова Н.В., Чернавский Д.С. Математическое моделирование в биофизике. - М. : Наука, 1975. - 344 с.

6. Романовский Ю.М., Сидорова Г.А. О влиянии диффузии на затухание колебательных химических реакций // Колебательные процессы в биологических и химических системах. - Труды всесоюзного симпозиума. - Пущино, 1966. - С. 258-266.

7. Марри Дж. Нелинейные дифференциальные уравнения в биологии. Лекции о моделях : пер. с англ. - М. : Мир, 1983. - 397 с.

8. Фарлоу С. Уравнения с частными производными для научных работников и инженеров : пер. с англ. М. : Мир, 1985. - 384 с.

9. Ландэ Д.В. Основы интеграции информационных потоков. - К. : Инжиниринг, 2006. - 240 с.

10. Додонов А.Г., Ландэ Д.В. Живучесть информационных систем. - К. : Наук. думка, 2011. - 256 с.

11. Бушуев А.Б. Математическое моделирование процессов технического творчества. - СПб : СПбГУ ИТМО, 2010. - 181 с.

12. Гвардейцев М.И., Кузнецов П.Г., Розенберг В.Я. Математическое обеспечение управления. Меры развития общества. - СПб. : Специальная литература, 2016. -222 c.

\section{REFERENCES}

1. Ivanov A.K. Razrabotka $i$ primenenie matematicheskikh modelei ierarkhicheskikh ASU [Development and Application of Mathematical Models of Hierarchical Computer-Aided Control Systems]. Ulyanovsk, UISTU Publ., 2017. 262 p.

2. Ivanov A.K., Beresnev lu.I. Dinamika raspredeleniia informatsii $v$ ierarkhicheskikh sistemakh [The Dynamics of Information Distribution in Hierarchical Systems]. Avtomatizatsiia protsessov upravleniia [Automation of Control Processes], 2017, no. 4 (50), pp. 25-35.

3. IvanovA.K. Matematicheskiemodeli informatsionnogo prostranstva ierarkhicheskikh sistem [Mathematical Models of Hierarchical Systems Information Space]. Avtomatizatsiia protsessov upravleniia [Automation of Control Processes], 2018, no. 1 (51), pp. 47-57.

4. Alekseev V.V. Fizicheskoe $i$ matematicheskoe modelirovanie ekosistem [Physical and Mathematical Modeling of Ecosystems]. St. Petersburg, Gidrometeoizdat Publ., 1992. 367 p.

5. Romanovskii lu.M., Stepanova N.V., Chernavskii D.S. Matematicheskoe modelirovanie $v$ biofizike [Mathematical Modeling in Biological Physics]. Moscow, Nauka Publ., 1975. 344 p.

6. Romanovskii lu.M., Sidorova G.A. O vliianii diffuzii na zatukhanie kolebatelnykh khimicheskikh reaktsii [On Diffusion Affecting to the Attenuation of an Oscillation Chemical Reaction]. Kolebatelnye protsessy $v$ biologicheskikh i khimicheskikh sistemakh. Trudy vsesoiuznogo simpoziuma [Oscillating in Biological and Chemical Systems. Proc. All-Soviet Union Symposium]. Pushchino, 1966, pp. 258-266.

7. Marri J. Nelineynye differentsialnye uravneniia $v$ biologii. Lektsii o modeliakh. Per. s angl. [Nonlinear Differential Equations in Biology. Transl. from Engl.]. Moscow, Mir Publ., 1983. 397 p.

8. Farlow S. Uravneniia s chastnymi proizvodnymi dlia nauchnykh rabotnikov i inzhenerov. Per. s angl. [Partial Differential Equations for Scientists and Engineers. Transl. from Engl.]. Moscow, Mir Publ., 1985. 384 p.

9. Lande D.V. Osnovy integratsii informatsionnykh potokov [Principles of Data Flow Integration]. Kiev, Engineering Pul., 2006. 240 p.

10. Dodonov A.G., Lande D.V. Zhivuchest informatsionnykh system [Information System Survivability]. Kiev, Nauk. Dumka Publ., 2011. 256 p.

11. Bushuev A.B. Matematicheskoe modelirovanie protsessov tekhnicheskogo tvorchestva [Mathematical Modelling of Technical Creativity Processes]. St. Petersburg, SPbGU ITMO Publ., 2010. 181 p.

12. Gvardeitsev M.I., Kuznetsov P.G., Rozenberg V.la. Matematicheskoe obespechenie upravleniia. Mery razvitiia obshchestva [Mathematical Support of Management. Social Development Measures]. St. Petersburg, Spetsialnaia Literatura Publ., 2016. 222 p. 